
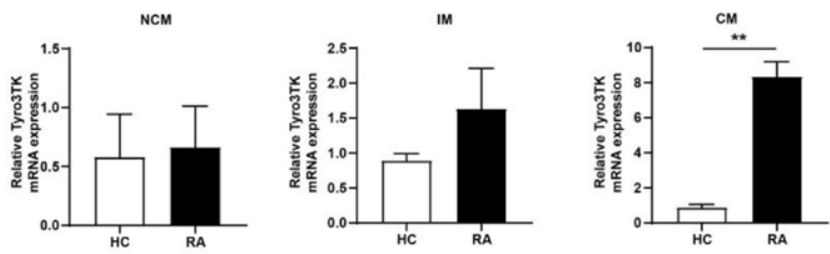

b
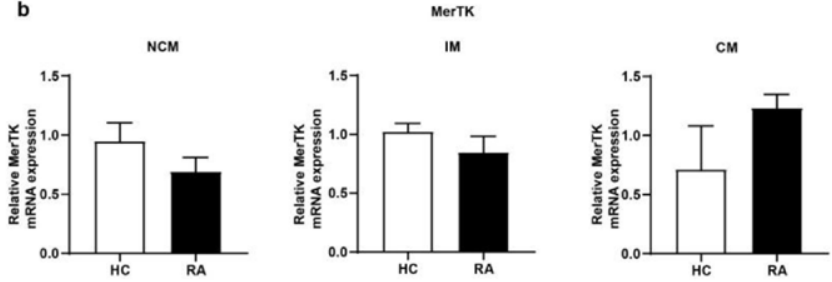

Figure 3. The mRNA expression of Tyro3TK on CM is increased in RA. (a) The mRNA expression of Tyro3TK on NCM, IM, and CM in HC $(n=3)$ and RA $(n=3)$ patients. (b) The mRNA expression of MerTK on NCM, IM, and CM in $\mathrm{HC}(\mathrm{n}=3)$ and RA $(\mathrm{n}=3)$ patients. ${ }^{* *} P$ $<0.01$.

Conclusion: These findings indicate that Tyro3TK on CM was elevated in RA patients and correlated positively with disease activity, which may serves as an important participant in RA pathogenesis.

REFERENCES:

[1] Rothlin CV, Lemke G, TAM receptor signaling and autoimmune disease. Curr Opin Immunol, 2010. 22(6): p. 740-6.

Footnotes: The study was supported by grants from the National Natural Science Foundation of China (81671609 and 81871290 to Dr. Y. Su, 82001718 to Dr. L. Xu), the Beijing Science and Technology Planning Project (Z191100006619111 to Dr. Y. Su), the Beijing Municipal Natural Science Foundation (7194329 to Dr. L. Xu).
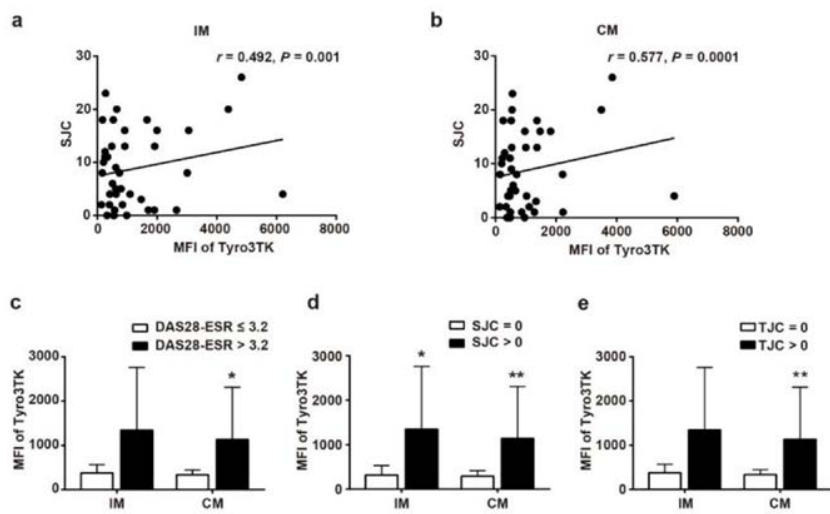

Figure 4. Correlation analysis of Tyro3TK on IM and CM with RA patient clinical manifestations. The associations of Tyro3TK on IM $\left(r=0.492,{ }^{\star} P=0.001\right)$ (a) and $\mathrm{CM}\left(\mathrm{r}=0.577,{ }^{\star} P=\right.$ 0.0001 ) (b) with RA patient swollen joint counts (SJC) were analyzed, respectively. The expression of Tyro3TK on IM and CM were also compared between different RA patient groups: (c) RA with high disease activity (DAS28-ESR $>3.2$ ) and non-high disease activity (DAS28-ESR $\leq$ 3.2), (d) RA with and without swollen joints, (e) tender joints. ${ }^{\star} P<0.05,{ }^{\star \star} P<0.01$

Disclosure of Interests: None declared DOI: 10.1136/annrheumdis-2021-eular.636

\section{AB0047 CLINICAL AND RADIOLOGICAL CHARACTERISTICS AND TREATMENTS OF A COHORT OF PATIENTS WITH RHEUMATOID ARTHRITIS AND DIFFUSE INTERSTICIAL PULMONARY DISEASE FOLLOWED AT THE VIRGEN MACARENA UNIVERSITY HOSPITAL}

P. Muñoz Reinoso ${ }^{1}$, F. J. Toyos Sáenz de Miera ${ }^{1}$, D. Ruiz-Montesinos ${ }^{1}$, I. García Hernández ${ }^{1}$, P. Guerrero Zamora ${ }^{2}$, J. J. Pérez Venegas ${ }^{1} .{ }^{1}$ Hospital Universitario Virgen Macarena, Reumatolog ía, Sevilla, Spain; ${ }^{2}$ Hospital Universitario Virgen Macarena, Neumología, Sevilla, Spain

Background: Diffuse interstitial lung disease (ILD) is an extra-articular manifestation of rheumatoid arthritis $(R A)^{1,2}$. The most common pattern is the usual interstitial pneumonia (UIP), conditioning a worse prognosis ${ }^{3}$.
Objectives: To describe epidemiological, clinical, and radiological characteristics and treatment used in a cohort of patients diagnosed with RA and ILD.

Methods: Retrospective descriptive study, including patients with a diagnosis of RA and ILD, reviewed in the Rheumatology and Pneumology consultations of the Virgen Macarena University Hospital, from 2010 to 2019. Data obtained from medical records are analyzed. SPSS statistical software is used.

Results: 26 patients were included, 18 women (69.2\%). Median age at diagnosis of ILD was 62 years (53-73). Twelve patients (46.6\%) used to smoke. The mean time from RA diagnosis to ILD diagnosis was 79 months (8-264). The RF was positive in $91.3 \%$ cases (21) and $87 \%$ of them (20) were ACPA positive. Fourteen patients $(53.8 \%)$ had erosions and $3(11.5 \%)$ had an associated Sjögren's Syndrome. When ILD was diagnosed, the RA activity by DAS28PCR was moderate (3.28; $2.34-3.28)$ and 13 patients $(54.1 \%)$ suffered from dyspnea. The mean value of FVC and DLCO in the first assessment was $84 \%$ (63-108) and 71.7\% (64-86), respectively. The most frequent radiological pattern of ILD was NINE in 15 patients $(57.7 \%), 6$ of them $(23.1 \%)$ had UIP and $5(19.3 \%)$ presented other patterns. Prior to ILD diagnosis, $24(92.3 \%)$ patients received oral glucocorticoids, $18(69.2 \%)$ cases started treatment with c-DMARD and 11 (42.3\%) of them with b-DMARD the most widely used were methotrexate (MTX) in 17 patients $(65.4 \%)$ and anti$\mathrm{TNF} \alpha$ in 10 (38.5\%). After diagnosis, treatment was changed to 12 patients $(46.6 \%)$; the most used DMARD was leflunomide, in $11(42.3 \%)$, MTX was maintained in 7 patients $(26.9 \%)$; the number of anti-TNF $\alpha$ used decreased to 4 cases $(15.4 \%)$, using instead drugs such as rituximab $5(19.2 \%)$, abatacept $3(11.5 \%)$ baricitinib $2(7.7 \%)$ and anti-IL6 2 (7, 7\%). During follow-up, 11 cases $(57.9 \%)$ remained radiologically stable. A slight deterioration in DLCO was observed $(66 \%$; 51-80) and there was one death due to lung disease (UIP).

Conclusion: In this study, the most frequent radiological pattern was NINE. Half of the patients used to smoke. At the diagnosis of ILD (at ILD diagnosis), dyspnea was the most relevant clinical symptom, with a slight deterioration in the? respiratory function tests. This represented a change in the therapeutic strategy. REFERENCES:

[1] Olson AL, Swigris JJ, Sprunger DB, et al. Rheumatoid arthritis-interstitial lung disease-associated mortality. Am J Respir Crit Care Med. 2011;183:372-78.

[2] Fragoulis GE, Nikiphorou E, Larsen J, Korsten P and Conway R. Methotrexate-Associated Pneumonitis and Rheumatoid Arthritis-Interstitial Lung Disease: Current Concepts for the Diagnosis and Treatment. Front. Med. 2019;6:238

[3] Tanaka N, Kim JS, Newell JD, et al. Rheumatoid arthritis-related lung diseases: CT findings. Radiology. 2004;232:81-91.

Disclosure of Interests: None declared

DOI: 10.1136/annrheumdis-2021-eular.1413

\section{AB0048 \\ ENZYMATIC PATTERN OF CIRCULATING PRO- AND ANTIOXIDANT ENZYMES IN RHEUMATOID ARTHRITIS PATIENTS}

S. Bedina ${ }^{1}$, E. Mozgovaya ${ }^{1}$, A. Trofimenko ${ }^{1}$, S. Spitsina ${ }^{1}$, M. Mamus ${ }^{1}$.

${ }^{1}$ Research Institute of Clinical and Experimental Rheumatology named after A.B. Zborovsky, Clinical Biochemistry Lab, Volgograd, Russian Federation

Background: Rheumatoid arthritis (RA) is an autoimmune rheumatic disease of unknown etiology characterized by chronic erosive arthritis and systemic organ involvement resulting in early disability and shorter life expectancy. Neutrophils are suggested to play a substantial role in the induction and promotion of autoimmune inflammation in RA. This ability can be based on newly discovered feature of neutrophils to release neutrophil extracellular traps (NETs) during specific type cell death called NETosis. Hyperproduction of reactive oxygen species (ROS) is one of the factors promoting NETs production. With this background, the study of pro- and antioxidant enzymatic activities in RA patients can be of great interest. Objectives: To assess plasma activities of essential prooxidant and antioxidant enzymes in RA patients.

Methods: The research was carried out in agreement with the WMA Declaration of Helsinki principles. 71 RA patients (46 women and 25 men) were enrolled in the study. The diagnosis was verified using ACR/EULAR criteria (2010). RA activity was measured using the Disease Activity Score of 28 joints (DAS28). 30 healthy persons comprise control group. Plasma xanthine oxidase (XO; EC 1.17.3.2), xanthine dehydrogenase $(X D H ; E C$ 1.17.1.4) and superoxide dismutase (SOD; EC 1.15.1.1) activities were measured using spectrophotometric technique. XO and XDG activities were expressed as $\mathrm{nmol} / \mathrm{ml} / \mathrm{min}$, SOD activity - as units of action. Statistical analysis was performed using Statistica 6.0 software package. Differences were considered significant when $p<0.05$. Reference ranges were calculated as means $\pm 2 S D$.

Results: Mean age of patients was $43.2 \pm 3.6$ years, mean RA duration was $11.9 \pm 2.6$ years. $24(33.8 \%)$ RA patients had low disease activity, and $6(8.5 \%)$ patients had high one. Extra-articular manifestations were found in $30(42.2 \%)$ patients. $30 \%$ of them had cardiovascular involvement, $23.3 \%$ - pulmonary lesions, and $23.3 \%$ had renal involvement. Reference ranges for XO, XDG, and SOD activities were 2.28-5.12 nmol $/ \mathrm{min} / \mathrm{ml}, 3,96-7,24 \mathrm{nmol} / \mathrm{min} / \mathrm{ml}$, and 3,136,58 units, respectively. We examined activities of these enzymes in circulation 
of RA patients with different patterns of clinical manifestations as well as relationship between RA activity and XO, XDG, and SOD activities. RA patients had increased both mean $X O$ and mean SOD activities ( $p<0.001$ for both enzymes). $\mathrm{XO}$ activity reached its highest values at maximum disease activity and overt extra-articular involvements, while SOD activity did it in moderate and high disease activities as well as in patients with joint manifestations. XDG activity was increased in low disease activity $\left(p^{<} 0.001\right)$ and solely joint lesions $(p=0.011)$, while moderate or high disease activities $(\mathrm{p}=0.008)$ and extra-articular involvements $(p=0.025)$ were characterized by decreased activity of this enzyme. Conclusion: We have revealed substantial multidirectional changes of plasma $\mathrm{XO}$ and XDG activities in RA. Plasma enzymatic pattern in RA patients is characterized by activation of both oxidant and antioxidant metabolic pathways. Activities of XO and SOD were positively correlated with RA activity, while XDG activity was negative correlated with RA activity. The differences between selective articular RA type and RA form with extraarticular manifestations were also revealed. Changes in oxidant and antioxidant enzyme activities can be connected with anticitrulline autoimmunity in RA via production of citrulline-rich neutrophil extracellular traps, thus enhancing rheumatoid autoimmunity.

Disclosure of Interests: None declared

DOI: 10.1136/annrheumdis-2021-eular.1754

\begin{tabular}{|l|l}
\hline AB0049 & RELATIONSHIP OF RHEUMATOID FACTOR AND \\
& XANTHINE OXIDOREDUCTASE ENZYMATIC \\
CONSTITUENTS IN SEVERAL BLOOD \\
COMPARTMENTS OF RHEUMATOID ARTHRITIC \\
PATIENTS
\end{tabular}

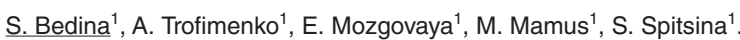
${ }^{1}$ Research Institute of Clinical and Experimental Rheumatology named after A.B. Zborovsky, Clinical Biochemistry Lab, Volgograd, Russian Federation

Background: Rheumatoid arthritis (RA) is a systemic autoimmune disease characterized by the presence of rheumatoid factor (RF) and anticitrulline autoantibodies. Recent evidences suggest that impairment of neutrophil extracellular traps (NETs) could exert substantial influence on RA pathogenesis. The production of NETs depends heavily on the ROS generation. One of its mechanisms is xanthine oxidoreductase (XOR) mediated degradation of purine metabolites. Analysis of pro-oxidant activity of the enzymatic complex XOR and its constituents, xanthine oxidase (XO) and xanthine dehydrogenase (XDG), is an issue of considerable interest in this context.

Objectives: Evaluation of XO and XDG activities in RF-positive and RF-negative RA using both plasma and lysed lymphocyte samples.

Methods: The research was carried out in agreement with the WMA Declaration of Helsinki principles. Diagnosis of RA had been verified using ACR/EULAR 2010 criteria. Enzymatic activities in plasma and lymphocytes were measured spectrophotometrically and expressed as $\mathrm{nmol} / \mathrm{min} / \mathrm{ml}$. Enzymatic activities in lymphocytes were also normalized to $1 \times 107 \mathrm{cells} / \mathrm{ml}$. Statististical tests were selected in line with common guidelines. Differences were considered significant when $p<0.05$. Reference ranges were calculated as means $\pm 2 S D$.

Results: 75 adult RA patients (52 females and 23 males, mean age $43.9 \pm 0.97$ years, mean disease duration $8.5 \pm 0.3$ years) from the rheumatology unit of Volgograd Clinical Emergency Hospital \#25 as well as 35 healthy controls were included in the study. RF-positive RA and RF-negative RA were observed in 49 (65.3\%) and $26(34.7 \%)$ patients, respectively. Reference ranges for plasma and lymphocyte XO activities were $2.60-3.96$ and $14.2-27.8 \mathrm{nmol} / \mathrm{min} / \mathrm{ml}$, respectively. Similar ranges for XDG activities were 4.49-5.93 and $22.5-40.7 \mathrm{nmol} / \mathrm{min} / \mathrm{ml}$, respectively. Enzymatic profile of RA patients is characterized by significantly increased $\mathrm{XO}$ activity in plasma and decreased $X O$ and XDG activities in lymphocytes ( $p^{<0.001) . X O}$ activity is increased $\left(p^{<} 0.001\right), X D G$ activity is decreased $\left(p^{<} 0.001\right)$ in blood plasma of patients with RF-negative RA, while the activity of both enzymes is decreased in lymphocytes $\left(p^{<0.001)}\right.$. XO activity $\left(p^{<0.001)}\right.$ and XDG activity $(p<0.05)$ is increased in blood plasma, XO activity and XDG activity are decreased $\left(p^{<0.001)}\right.$ in lymphocytes of patients with RF-positive RA. Plasma XO and XDG activities are also higher, and lymphocyte $\mathrm{XO}$ and XDG activities are lower in patients with RF-positive RA than in patients with RF-negative RA ( $p<0.001)$.

Conclusion: Our study revealed the relationship between enzyme parameters and rheumatoid factor presence. More pronounced changes in the enzyme activities were observed in patients with RF-positive RA. These results demonstrate that activation of the xanthine oxidase/xanthine dehydrogenase enzyme complex is an substantial factor of induction and continuation of the autoimmune rheumatoid inflammation.

Disclosure of Interests: None declared

DOI: 10.1136/annrheumdis-2021-eular.1756

\section{AB0050 \\ A NOVEL METHOD FOR ISOLATION OF EXOSOMES FROM SYNOVIAL FLUID}

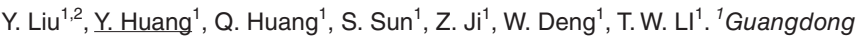

Second Provincial General Hospital, Department of Rheumatology and
Immunology, Guangzhou, China; ${ }^{2}$ University of South China, Guangdong Second Provincial General Hospital, Guangzhou, China

Background: Exosomes in synovial fluid (SF) has a close relationship with the pathogenesis of rheumatiod arthritis. As a complex biological fluid, SF presents challenges for exosomes isolation using standard methods, such as Exoquick ${ }^{\mathrm{TM}}$ kit and ultracentrifugation.

Objectives: The study aims to compared the quality of exosomes separated by

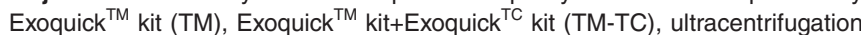
(UC) and TM-TC+UC(TM-TC-UC) from SF.

Methods: Exosomes was separated by TM, TM-TC, UC and TM-TC-UC respectively. The size and concentrations of exosomes were detected by high sensitivity flow cytometry for nanoparticle analysis. Total protein and RNA were extracted from exosomes. SDS-PAGE was used to detect the protein distribution of exosomes. Western blot was used to examine the level of albumin and exosomes marker (TSG101 and CD81).

Results: There was no statistic difference in the diameters of exosomes separated by the four methods. The concentrations of exosomes in TM, TM-TC TM-TC-UC and UC were (5.65 \pm 0.93$),(3.02 \pm 1.19),(1.67 \pm 0.25)$ and $(4.61 \pm 0.73)$ ${ }^{*} 10^{9} \mathrm{Particles} / \mathrm{mL}$. The protein concentrations of exosomes separated by the four methods were consistent with the concentrations of exosomes. SDS-PAGE showed that the protein distribution of exosomes separated by the four methods were different. Low levels of albumin were detected in TM-TC and TM-TC-UC, while high levels of albumin in TM and UC. Total RNA concentrations from exosomes in TM-TC was higher than other groups.

Conclusion: TM-TC can be used to obtain higher quality exosomes from SF for the study of exosome-enriched components.

REFERENCES:

[1] Helwa I, et al, A Comparative Study of Serum Exosome Isolation Using Differential Ultracentrifugation and Three Commercial Reagents. PloS one, 2017. 12(1): p. e0170628-e0170628

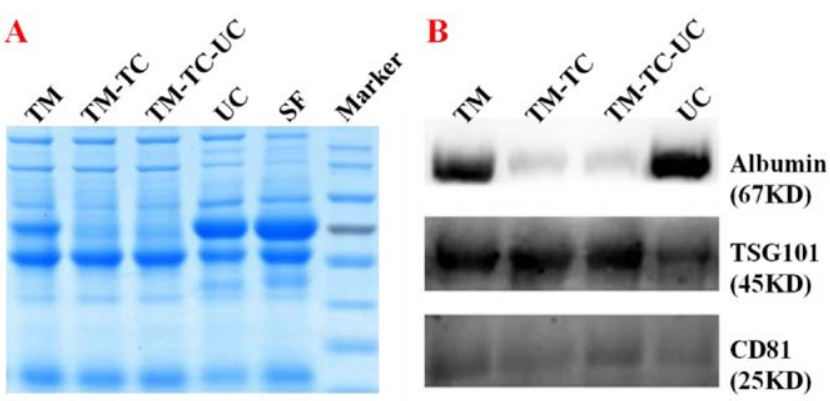

Figure 1. A: SDS-PAGE showed the protein distribution of exosomes; $\mathbf{B}$ : the detection of albumin, TSG101 and CD81 by western blot.

Disclosure of Interests: None declared

DOI: 10.1136/annrheumdis-2021-eular.1996

\begin{tabular}{|l}
\hline AB0051 \\
THE EFFECTS OF NON-INVASIVE VAGUS NERVE \\
STIMULATION ON IMMUNOLOGICAL RESPONSES \\
AND PATIENT REPORTED OUTCOME MEASURES \\
OF FATIGUE IN PATIENTS WITH CHRONIC FATIGUE \\
SYNDROME, FIBROMYALGIA, AND RHEUMATOID \\
ARTHRITIS
\end{tabular}

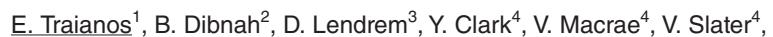
K. Wood ${ }^{1}$, D. Storey ${ }^{1}$, B. Simon ${ }^{5}$, J. Blake ${ }^{5}$, J. Tarn ${ }^{3}$, W. F. Ng ${ }^{3,4} \cdot{ }^{1}$ Newcastle University, FMS Professional Services, Newcastle upon Tyne, United Kingdom; ${ }^{2}$ University of Glasgow, Institute of Molecular Cell \& Systems Biology, Glasgow, United Kingdom; ${ }^{3}$ Newcastle University, Translational \& Clinical Research, Newcastle upon Tyne, United Kingdom; ${ }^{4}$ National Institute for Health Research (NIHR), Newcastle Biomedical Research Centre (BRC), Newcastle upon Tyne, United Kingdom; ${ }^{5}$ electroCore, Inc., NJ, United States of America

Background: Fatigue is reported as a common symptom among autoimmune and other chronic diseases such as fibromyalgia (FM), a long-term condition with uncertain pathophysiology. Previous studies from our group suggest that non-invasive vagus nerve stimulation ( $\mathrm{nVNS}$ ) may contribute to the improvement of patient reported outcome measures (PROMs) of fatigue in patients with primary Sjögren's Syndrome (1)

Objectives: This follow-up study uses the gammaCore device (electroCore) to assess the effect of nVNS on PROMs of fatigue and immune responses in chronic fatigue syndrome (CFS), FM and rheumatoid arthritis (RA).

Methods: The study included thirteen CFS, fourteen FM and fifteen RA patients who used the gammaCore nVNS device twice daily over a 26-day period. Preand post- nVNS bloods were drawn at baseline and final visits. Whole blood 